Supporting Information for:

\title{
A Charge-Transfer-Induced Spin-Transition in a Discrete Complex: The Role of Extrinsic Factors in Stabilizing Three Electronic Isomeric Forms of a Cyanide-Bridged Co/Fe Cluster
}

Curtis P. Berlinguette, Alina Dragulescu-Andrasi, ${ }^{\dagger}$ Andreas Sieber, ${ }^{\dagger}$ Hans-Ulrich

\author{
Güdel, ${ }^{\ddagger}$ Catalina Achim, ${ }^{* \dagger}{ }^{\dagger}$ Kim R. Dunbar*
}

[*] Dr. K. R. Dunbar

Department of Chemistry, Texas A\&M University

PO Box 30012, College Station, Texas 77842-3012 (USA)

Phone: (979) 845-5235

Fax: (979) 845-7177

E-mail: dunbar@chem.mail.tamu.edu

[†] Department of Chemistry, Carnegie Mellon University, 4400 Fifth Avenue, Pittsburgh, PA 15213.

[\$] Department of Chemistry and Biochemistry, University of Bern, Freiestrasse 3, 3000 Bern 9, Switzerland 
Table S1. Crystallographic data and structural refinement parameters for separate crystals of $\left\{\left[\mathrm{Co}(\operatorname{tmphen})_{2}\right]_{3}\left[\mathrm{Fe}(\mathrm{CN})_{6}\right]_{2}\right\}(\mathbf{1})$ over the 30 298 temperature range $\left(\mathbf{1}^{\mathbf{a}}-\mathbf{1}^{\mathbf{i}}\right)$.

\begin{tabular}{|c|c|c|c|c|}
\hline & $\left(1^{a}\right) \cdot 9 \mathrm{H}_{2} \mathrm{O}$ & $\left(\mathbf{1}^{\mathbf{b}}\right) \cdot 12 \mathrm{H}_{2} \mathrm{O}$ & $\left(\mathbf{1}^{\mathrm{c}}\right) \cdot 13 \mathrm{H}_{2} \mathrm{O}$ & $\left(\mathbf{1}^{\mathbf{d}}\right) \cdot 11 \mathrm{H}_{2} \mathrm{O}$ \\
\hline Chemical formula & $\mathrm{C}_{108} \mathrm{H}_{114} \mathrm{Co}_{3} \mathrm{Fe}_{2} \mathrm{~N}_{24} \mathrm{O}_{9}$ & $\mathrm{C}_{108} \mathrm{H}_{120} \mathrm{Co}_{3} \mathrm{Fe}_{2} \mathrm{~N}_{24} \mathrm{O}_{12}$ & $\mathrm{C}_{108} \mathrm{H}_{122} \mathrm{Co}_{3} \mathrm{Fe}_{2} \mathrm{~N}_{24} \mathrm{O}_{13}$ & $\mathrm{C}_{108} \mathrm{H}_{118} \mathrm{Co}_{3} \mathrm{Fe}_{2} \mathrm{~N}_{24} \mathrm{O}_{11}$ \\
\hline$T(\mathrm{~K})$ & $30(2)$ & $90(2)$ & $110(2)$ & $130(2)$ \\
\hline$a(\AA)$ & $18.94(1)$ & $19.04(4)$ & $19.02(1)$ & $19.05(1)$ \\
\hline$b(\AA)$ & $24.79(2)$ & $24.77(5)$ & $25.03(2)$ & $24.95(2)$ \\
\hline$c(\AA)$ & $24.33(1)$ & $24.56(5)$ & $24.65(2)$ & $24.782(2)$ \\
\hline$\beta\left(^{\circ}\right)$ & $97.77(9)$ & $97.72(9)$ & $97.86(2)$ & $97.69(2)$ \\
\hline$V\left(\AA^{3}\right)$ & $11319(12)$ & $11487(42)$ & $11626(15)$ & $11676(15)$ \\
\hline$Z$ & \multicolumn{4}{|c|}{4} \\
\hline formula weight & 2181.71 & 2234.75 & 2252.77 & 2216.7 \\
\hline space group & \multicolumn{4}{|c|}{$P 2_{1} / \mathrm{c}($ No. 14$)$} \\
\hline$D_{\text {calc }}\left(\mathrm{g} \mathrm{cm}^{-3}\right)$ & 1.269 & 1.279 & 1.272 & 1.248 \\
\hline$\mu\left(\mathrm{mm}^{-1}\right)$ & 0.745 & 0.738 & 0.730 & 0.724 \\
\hline$\theta$ range $\left(^{\circ}\right)$ & $1.36-20.82$ & $1.84-20.81$ & $1.63-20.81$ & $1.08-20.82$ \\
\hline$G O F\left(\mathrm{~F}^{2}\right)$ & 1.094 & 1.043 & 1.035 & 1.136 \\
\hline Data / parameters ${ }^{\mathrm{n}}$ & 11827 / 1371 & $12006 / 1387$ & $12158 / 1399$ & $12224 / 1395$ \\
\hline$R\left(F_{o}\right)^{\mathrm{p}^{\mathrm{t}}}$ & 0.1255 & 0.0835 & 0.0760 & 0.0971 \\
\hline$R_{w}\left(F_{o}^{2}\right)^{\mathrm{q}}$ & $0.3021^{\mathrm{r}}$ & $0.2368^{\mathrm{s}}$ & $0.2058^{\mathrm{t}}$ & $0.2587^{\mathrm{u}}$ \\
\hline
\end{tabular}


Table S1 (cont'd).

\begin{tabular}{|c|c|c|c|c|}
\hline & $\left(\mathbf{1}^{\mathbf{e}}\right) \cdot 8 \mathrm{H}_{2} \mathrm{O}$ & $\left(\mathbf{1}^{\mathrm{f}}\right) \cdot 7 \mathrm{H}_{2} \mathrm{O}$ & $\left(\mathbf{1}^{\mathbf{g}}\right) \cdot 8 \mathrm{H}_{2} \mathrm{O}$ & $\left(\mathbf{1}^{\mathbf{h}}\right) \cdot 9 \mathrm{H}_{2} \mathrm{O}$ \\
\hline Chemical formula & $\mathrm{C}_{108} \mathrm{H}_{112} \mathrm{Co}_{3} \mathrm{Fe}_{2} \mathrm{~N}_{24} \mathrm{O}_{8}$ & $\mathrm{C}_{108} \mathrm{H}_{110} \mathrm{Co}_{3} \mathrm{Fe}_{2} \mathrm{~N}_{24} \mathrm{O}_{7}$ & $\mathrm{C}_{108} \mathrm{H}_{112} \mathrm{Co}_{3} \mathrm{Fe}_{2} \mathrm{~N}_{24} \mathrm{O}_{8}$ & $\mathrm{C}_{108} \mathrm{H}_{112} \mathrm{Co}_{3} \mathrm{Fe}_{2} \mathrm{~N}_{24} \mathrm{O}_{8}$ \\
\hline$T(\mathrm{~K})$ & $150(2)$ & $170(2)$ & $190(2)$ & $220(2)$ \\
\hline$a(\AA)$ & $19.05(5)$ & $19.09(2)$ & $19.141(4)$ & $19.261(4)$ \\
\hline$b(\AA)$ & $25.16(7)$ & $25.21(3)$ & $25.099(5)$ & $25.123(5)$ \\
\hline$c(\AA)$ & $24.45(7)$ & $24.69(2)$ & $24.698(5)$ & $24.782(5)$ \\
\hline$\beta\left(^{\circ}\right)$ & $97.62(6)$ & $97.67(4)$ & $97.54(3)$ & $97.74(3)$ \\
\hline$V\left(\AA^{3}\right)$ & $11617(54)$ & $11779(21)$ & $11763(4)$ & $11883(4)$ \\
\hline$Z$ & & & 4 & \\
\hline $\begin{array}{l}\text { formula weight } \\
\text { space group }\end{array}$ & 2162.7 & 2144.7 & $\begin{array}{l}2162.7 \\
\text { (No. 14) }\end{array}$ & 2176.3 \\
\hline$D_{\text {calc }}\left(\mathrm{g} \mathrm{cm}^{-3}\right)$ & 1.227 & 1.201 & 1.212 & 1.209 \\
\hline$\mu\left(\mathrm{mm}^{-1}\right)$ & 0.725 & 0.713 & 0.716 & 0.709 \\
\hline$\theta$ range $\left(^{\circ}\right)$ & $1.35-20.81$ & $2.03-20.81$ & $2.03-20.81$ & $1.62-20.81$ \\
\hline$G O F\left(\mathrm{~F}^{2}\right)$ & 0.989 & 1.013 & 1.029 & 1.034 \\
\hline Data / parameters ${ }^{\mathrm{n}}$ & $12002 / 1326$ & $12281 / 135$ & $12307 / 1363$ & 12433 / 1379 \\
\hline$R\left(F_{o}\right)^{\mathrm{p}}$ & 0.1210 & 0.0954 & 0.0851 & 0.0765 \\
\hline$R_{w}\left(F_{o}^{2}\right)^{\mathrm{r}}$ & $0.2919^{\mathrm{v}}$ & $0.2664^{\mathrm{w}}$ & $0.2446^{\mathrm{x}}$ & $0.2234^{\mathrm{y}}$ \\
\hline
\end{tabular}


Table S1 (cont'd).

\begin{tabular}{|c|c|}
\hline & $\left(\mathbf{1}^{\mathrm{i}}\right) \cdot 6 \mathrm{H}_{2} \mathrm{O}$ \\
\hline Chemical formula & $\mathrm{C}_{108} \mathrm{H}_{108} \mathrm{Co}_{3} \mathrm{Fe}_{2} \mathrm{~N}_{24} \mathrm{O}_{6}$ \\
\hline$T(\mathrm{~K})$ & $293(2)$ \\
\hline$a(\AA)$ & $19.04(2)$ \\
\hline$b(\AA)$ & 24.91(3) \\
\hline$c(\AA)$ & $24.62(4)$ \\
\hline$\beta\left(^{\circ}\right)$ & $97.64(8)$ \\
\hline$V\left(\AA^{3}\right)$ & $11569(25)$ \\
\hline Z & 4 \\
\hline formula weight & 2208.67 \\
\hline space group & $P 2_{1} / \mathrm{c}($ No. 14$)$ \\
\hline$D_{\text {calc }}\left(\mathrm{g} \mathrm{cm}^{-3}\right)$ & 1.214 \\
\hline$\mu\left(\mathrm{mm}^{-1}\right)$ & 0.725 \\
\hline$\theta$ range $\left(^{\circ}\right)$ & $1.51-20.81$ \\
\hline$G O F\left(\mathrm{~F}^{2}\right)$ & 1.035 \\
\hline Data / parameters ${ }^{\mathrm{n}}$ & 12078 / 1331 \\
\hline$R\left(F_{o}\right)^{\mathrm{p}}$ & 0.0735 \\
\hline$R_{w}\left(F_{o}^{2}\right)^{\mathrm{q}}$ & $0.2089^{\mathrm{z}}$ \\
\hline \multicolumn{2}{|c|}{ 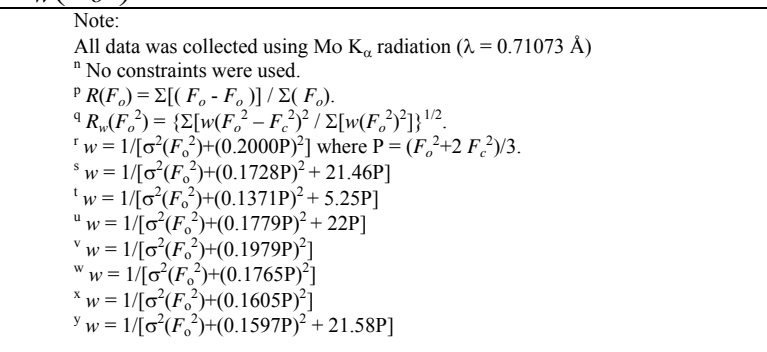 } \\
\hline${ }^{2} w=1 /\left[\sigma^{2}\left(F_{0}{ }^{2}\right)+(0.1420 P)^{2}\right]$ & \\
\hline
\end{tabular}


Table S2. Crystallographic data and structural refinement parameters for selected crystals of $\left\{\left[\mathrm{Co}(\mathrm{tmphen})_{2}\right]_{3}\left[\mathrm{Fe}(\mathrm{CN})_{6}\right]_{2}\right\}(\mathbf{1})$ after removing the weak reflections due to disordered solvent with the SQUEEZE program (PLATON) at $T=30 \mathrm{~K}\left(\mathbf{1}^{\mathbf{a}}\right), 110(2) \mathrm{K}$ $\left(\mathbf{1}^{\mathbf{b}}\right), 220(2) \mathrm{K}\left(\mathbf{1}^{\mathbf{g}}\right)$, and 293(2) K (1 $\left.\mathbf{1}^{\mathbf{h}}\right)$.

\begin{tabular}{|c|c|c|c|c|}
\hline & $\left(1^{\mathrm{a}}\right) \cdot 1 / 4 \mathrm{H}_{2} \mathrm{O}$ & $\left(\mathbf{1}^{\mathbf{b}}\right) \cdot 1 / 2 \mathrm{H}_{2} \mathrm{O}$ & $\left(1^{\mathrm{g}}\right)$ & $\left(1^{h}\right)$ \\
\hline Chemical formula & $\mathrm{C}_{108} \mathrm{H}_{96.5} \mathrm{Co}_{3} \mathrm{Fe}_{2} \mathrm{~N}_{24} \mathrm{O}_{0.25}$ & $\mathrm{C}_{108} \mathrm{H}_{97} \mathrm{Co}_{3} \mathrm{Fe}_{2} \mathrm{~N}_{24} \mathrm{O}_{0.5}$ & $\mathrm{C}_{108} \mathrm{H}_{96} \mathrm{Co}_{3} \mathrm{Fe}_{2} \mathrm{~N}_{24}$ & $\mathrm{C}_{108} \mathrm{H}_{96} \mathrm{Co}_{3} \mathrm{Fe}_{2} \mathrm{~N}_{24}$ \\
\hline$a(\AA)$ & $18.94(1)$ & $19.02(1)$ & $19.261(4)$ & $19.04(2)$ \\
\hline$b(\AA)$ & $24.79(2)$ & $25.03(2)$ & $25.123(5)$ & $24.91(3)$ \\
\hline$c(\AA)$ & $24.33(1)$ & $24.65(2)$ & $24.782(5)$ & $24.62(4)$ \\
\hline$\alpha\left(^{\circ}\right)$ & 90 & 90 & 90 & 90 \\
\hline$\beta\left({ }^{\circ}\right)$ & $97.77(9)$ & $97.86(2)$ & $97.74(2)$ & $97.64(8)$ \\
\hline$\gamma\left({ }^{\circ}\right)$ & 90 & 90 & 90 & 90 \\
\hline$V\left(\AA^{3}\right)$ & $11570(10)$ & $11630(20)$ & $11883(4)$ & $11569(25)$ \\
\hline Z & 4 & 4 & 4 & 4 \\
\hline formula weight & 2023.7 & 2027.6 & 2018.6 & 2018.6 \\
\hline space group & $P 2_{1} / \mathrm{c}($ No. 14$)$ & $P 2_{1} / \mathrm{c}($ No. 14$)$ & $P 2_{1} / \mathrm{c}($ No. 14$)$ & $P 2_{1} / \mathrm{c}($ No. 14$)$ \\
\hline$T(\mathrm{~K})$ & $30(2)$ & $110(2)$ & $220(2)$ & 293(2) \\
\hline$D_{\text {calc }}\left(\mathrm{g} \mathrm{cm}^{-3}\right)$ & 1.187 & 1.158 & 1.128 & 1.159 \\
\hline$\mu\left(\mathrm{mm}^{-1}\right)$ & 0.735 & 0.716 & 0.700 & 0.719 \\
\hline$\theta$ range $\left(^{\circ}\right)$ & $1.36-20.82$ & $1.63-20.81$ & $1.62-20.81$ & $1.51-20.81$ \\
\hline $\operatorname{GOF}\left(\mathrm{F}^{2}\right)$ & 0.925 & 0.916 & 0.975 & 0.910 \\
\hline Data / parameters ${ }^{t}$ & $11827 / 1263$ & $12158 / 1262$ & $12433 / 1258$ & $12078 / 1258$ \\
\hline$R\left(F_{o}\right)^{\mathrm{u}}$ & 0.0984 & 0.0550 & 0.0596 & 0.0578 \\
\hline$R_{w}\left(F_{o}^{2}\right)^{\mathrm{v}}$ & $0.2238^{\mathrm{w}}$ & $0.1284^{\mathrm{x}}$ & $0.1594^{y}$ & $0.1398^{\mathrm{z}}$ \\
\hline
\end{tabular}

\footnotetext{
No constraints were used.

${ }^{\mathrm{u}} R\left(F_{o}\right)=\Sigma\left[\left(F_{o}-F_{o}\right)\right] / \Sigma\left(F_{o}\right)$.

${ }^{\mathrm{v}} R_{w}\left(F_{o}^{2}\right)=\left\{\Sigma\left[w\left(F_{o}^{2}-F_{c}^{2}\right)^{2} / \Sigma\left[w\left(F_{o}^{2}\right)^{2}\right]\right\}^{1 / 2}\right.$

${ }^{\mathrm{w}} w=1 /\left[\sigma^{2}\left(\mathrm{Fo}^{2}\right)+(0.1535 \mathrm{P})^{2}\right]$ where $\mathrm{P}=\left(F_{o}{ }^{2}+2 F_{c}^{2}\right) / 3$

$w=1 /\left[\sigma^{2}\left(\mathrm{Fo}^{2}\right)+(0.0896 \mathrm{P})^{2}+5.25 \mathrm{P}\right]$ where $\mathrm{P}=\left(F_{o}^{2}+2 F_{c}^{2}\right) / 3$

$w=1 /\left[\sigma^{2}\left(\mathrm{Fo}^{2}\right)+(0.1151 \mathrm{P})^{2}\right]$ where $\mathrm{P}=\left(F_{o}^{2}+2 F_{c}^{2}\right) / 3$

${ }^{\mathrm{z}} w=1 /\left[\sigma^{2}\left(\mathrm{Fo}^{2}\right)+(0.1816 \mathrm{P})^{2}\right]$ where $\mathrm{P}=\left(F_{o}^{2}+2 F_{c}^{2}\right) / 3$
} 
Table S3. Selected bond distances $(\AA)$ for 1 at selected temperatures over the $30-293$ K temperature range.

\begin{tabular}{lllll}
\hline \multicolumn{1}{c}{$\begin{array}{c}\text { Connectivity } \\
\text { (Temperature })\end{array}$} & \multicolumn{1}{c}{$\mathbf{1}^{\mathbf{a}}$} & \multicolumn{1}{c}{$\mathbf{1}^{\mathbf{c}}$} & \multicolumn{1}{c}{$\mathbf{1}^{\mathbf{h}}$} & \multicolumn{1}{c}{$\mathbf{1}^{\mathbf{i}}$} \\
$(110 \mathrm{~K})$ & $(220 \mathrm{~K})$ & $(293 \mathrm{~K})$ \\
\hline $\mathrm{Co}(1)-\mathrm{N}(4)$ & $1.999(7)$ & $2.028(8)$ & $2.059(7)$ & $2.031(9)$ \\
$\mathrm{Co}(1)-\mathrm{N}(7)$ & $2.062(7)$ & $2.069(10)$ & $2.099(9)$ & $2.042(9)$ \\
$\mathrm{Co}(1)-\mathrm{N}(13)$ & $2.106(7)$ & $2.139(8)$ & $2.154(7)$ & $2.135(8)$ \\
$\mathrm{Co}(1)-\mathrm{N}(14)$ & $2.130(6)$ & $2.167(8)$ & $2.154(7)$ & $2.122(8)$ \\
$\mathrm{Co}(1)-\mathrm{N}(15)$ & $2.157(7)$ & $2.177(8)$ & $2.177(7)$ & $2.133(7)$ \\
$\mathrm{Co}(1)-\mathrm{N}(16)$ & $2.068(8)$ & $2.136(7)$ & $2.151(7)$ & $2.146(7)$ \\
$\mathrm{Co}(2)-\mathrm{N}(5)$ & $1.924(8)$ & $1.915(9)$ & $2.040(9)$ & $2.042(9)$ \\
$\mathrm{Co}(2)-\mathrm{N}(8)$ & $1.947(6)$ & $1.948(9)$ & $2.035(8)$ & $2.035(9)$ \\
$\mathrm{Co}(2)-\mathrm{N}(17)$ & $2.027(7)$ & $1.987(7)$ & $2.110(7)$ & $2.131(8)$ \\
$\mathrm{Co}(2)-\mathrm{N}(18)$ & $1.948(8)$ & $1.995(8)$ & $2.125(7)$ & $2.145(8)$ \\
$\mathrm{Co}(2)-\mathrm{N}(19)$ & $1.972(8)$ & $1.979(9)$ & $2.124(9)$ & $2.135(9)$ \\
$\mathrm{Co}(2)-\mathrm{N}(20)$ & $1.987(7)$ & $1.982(8)$ & $2.103(7)$ & $2.086(8)$ \\
$\mathrm{Co}(3)-\mathrm{N}(6)$ & $1.977(7)$ & $1.956(9)$ & $2.056(8)$ & $2.087(9)$ \\
$\mathrm{Co}(3)-\mathrm{N}(9)$ & $1.920(7)$ & $1.939(8)$ & $2.010(8)$ & $2.016(9)$ \\
$\mathrm{Co}(3)-\mathrm{N}(21)$ & $2.010(6)$ & $2.016(7)$ & $2.114(6)$ & $2.115(8)$ \\
$\mathrm{Co}(3)-\mathrm{N}(22)$ & $2.037(6)$ & $2.038(7)$ & $2.144(7)$ & $2.165(8)$ \\
$\mathrm{Co}(3)-\mathrm{N}(23)$ & $1.965(7)$ & $1.997(7)$ & $2.114(7)$ & $2.136(8)$ \\
$\mathrm{Co}(3)-\mathrm{N}(24)$ & $1.987(7)$ & $1.992(8)$ & $2.116(7)$ & $2.103(8)$ \\
$\mathrm{Fe}(1)-\mathrm{C}(1)$ & $1.92(1)$ & $1.95(1)$ & $1.93(1)$ & $1.90(2)$ \\
$\mathrm{Fe}(1)-\mathrm{C}(2)$ & $1.86(1)$ & $1.93(1)$ & $1.92(1)$ & $1.92(10$ \\
$\mathrm{Fe}(1)-\mathrm{C}(3)$ & $1.91(1)$ & $1.93(1)$ & $1.95(1)$ & $1.93(1)$ \\
$\mathrm{Fe}(1)-\mathrm{C}(4)$ & $1.908(9)$ & $1.90(1)$ & $1.942(9)$ & $1.92(1)$ \\
$\mathrm{Fe}(1)-\mathrm{C}(5)$ & $1.89(1)$ & $1.90(1)$ & $1.93(1)$ & $1.91(1)$ \\
$\mathrm{Fe}(1)-\mathrm{C}(6)$ & $1.901(8)$ & $1.94(1)$ & $1.93(1)$ & $1.89(1)$ \\
$\mathrm{Fe}(2)-\mathrm{C}(7)$ & $1.879(8)$ & $1.90(1)$ & $1.93(1)$ & $1.94(1)$ \\
$\mathrm{Fe}(2)-\mathrm{C}(8)$ & $1.882(8)$ & $1.90(1)$ & $1.92(1)$ & $1.91(1)$ \\
$\mathrm{Fe}(2)-\mathrm{C}(9)$ & $1.866(9)$ & $1.89(1)$ & $1.91(1)$ & $1.91(1)$ \\
$\mathrm{Fe}(2)-\mathrm{C}(10)$ & $1.898(9)$ & $1.92(1)$ & $1.92(1)$ & $1.88(1)$ \\
$\mathrm{Fe}(2)-\mathrm{C}(11)$ & $1.92(1)$ & $1.97(1)$ & $1.92(1)$ & $1.95(1)$ \\
$\mathrm{Fe}(2)-\mathrm{C}(12)$ & $1.86(1)$ & $1.91(1)$ & $1.89(1)$ & $1.88(1)$ \\
\hline & & & &
\end{tabular}

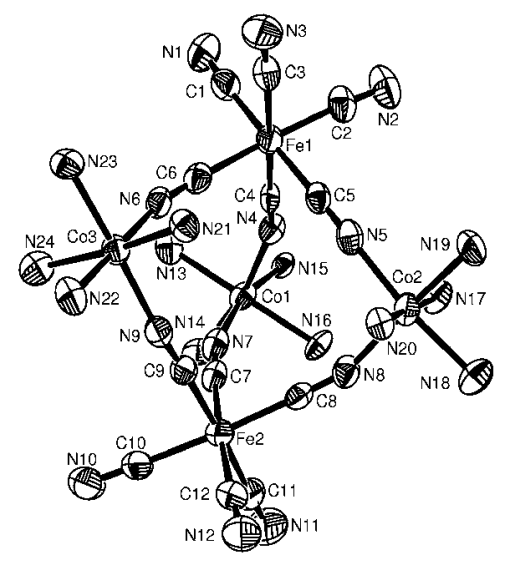


Table S4. Selected angles $\left({ }^{\circ}\right)$ for $\mathbf{1}$ before $\left(\mathbf{1}^{\mathrm{c}}\right)$ and after $\left(\mathbf{1}^{\mathrm{i}}\right)$ the onset of the structural transformation at the $\mathrm{Co}(2)$ and $\mathrm{Co}(3)$ centers. See Table S4 for atom labels.

\begin{tabular}{|c|c|c|c|c|c|}
\hline $\begin{array}{l}\text { Connectivity } \\
\text { (Temperature ) }\end{array}$ & $\begin{array}{c}\mathbf{1}^{\mathbf{c}} \\
(110 \mathrm{~K}) \\
\end{array}$ & $\begin{array}{c}\mathbf{1}^{\mathbf{i}} \\
(293 \mathrm{~K}) \\
\end{array}$ & Connectivity & $\begin{array}{c}\mathbf{1}^{\mathbf{c}} \\
(110 \mathrm{~K}) \\
\end{array}$ & $\begin{array}{c}\mathbf{1}^{\mathbf{i}} \\
(293 \mathrm{~K}) \\
\end{array}$ \\
\hline $\mathrm{N}(4)-\mathrm{Co}(1)-\mathrm{N}(7)$ & $91.3(3)$ & $91.7(3)$ & $\mathrm{N}(7)-\mathrm{Co}(1)-\mathrm{N}(15)$ & $165.9(3)$ & $166.1(3)$ \\
\hline $\mathrm{N}(4)-\mathrm{Co}(1)-\mathrm{N}(13)$ & $91.1(3)$ & $90.5(3)$ & $\mathrm{N}(7)-\mathrm{Co}(1)-\mathrm{N}(16)$ & $90.8(3)$ & $91.4(3)$ \\
\hline $\mathrm{N}(4)-\mathrm{Co}(1)-\mathrm{N}(14)$ & $167.9(3)$ & $167.7(3)$ & $\mathrm{N}(14)-\mathrm{Co}(1)-\mathrm{N}(15)$ & $84.7(3)$ & $85.3(3)$ \\
\hline $\mathrm{N}(4)-\mathrm{Co}(1)-\mathrm{N}(15)$ & $95.1(3)$ & $94.9(3)$ & $\mathrm{N}(14)-\mathrm{Co}(1)-\mathrm{N}(13)$ & $76.9(3)$ & $77.2(3)$ \\
\hline $\mathrm{N}(4)-\mathrm{Co}(1)-\mathrm{N}(16)$ & $97.4(3)$ & $97.2(3)$ & $\mathrm{N}(15)-\mathrm{Co}(1)-\mathrm{N}(13)$ & $91.5(3)$ & $93.9(3)$ \\
\hline $\mathrm{N}(7)-\mathrm{Co}(1)-\mathrm{N}(13)$ & $101.0(3)$ & $98.2(3)$ & $\mathrm{N}(13)-\mathrm{Co}(1)-\mathrm{N}(16)$ & $165.3(3)$ & $167.5(3)$ \\
\hline $\mathrm{N}(7)-\mathrm{Co}(1)-\mathrm{N}(14)$ & $91.7(3)$ & $90.8(3)$ & $\mathrm{N}(14)-\mathrm{Co}(1)-\mathrm{N}(16)$ & $94.3(3)$ & $94.8(3)$ \\
\hline $\mathrm{N}(5)-\mathrm{Co}(2)-\mathrm{N}(17)$ & $91.3(3)$ & $91.6(3)$ & $\mathrm{N}(15)-\mathrm{Co}(1)-\mathrm{N}(16)$ & $75.9(3)$ & $75.7(3)$ \\
\hline $\mathrm{N}(5)-\mathrm{Co}(2)-\mathrm{N}(18)$ & 171.3(3) & $165.9(3)$ & $\mathrm{N}(8)-\mathrm{Co}(2)-\mathrm{N}(20)$ & $91.7(3)$ & 93.1(3) \\
\hline $\mathrm{N}(5)-\mathrm{Co}(2)-\mathrm{N}(19)$ & $89.8(3)$ & $89.3(3)$ & $\mathrm{N}(17)-\mathrm{Co}(2)-\mathrm{N}(19)$ & $94.3(4)$ & $96.6(4)$ \\
\hline $\mathrm{N}(5)-\mathrm{Co}(2)-\mathrm{N}(20)$ & $92.1(3)$ & $93.0(3)$ & $\mathrm{N}(17)-\mathrm{Co}(2)-\mathrm{N}(18)$ & $81.7(3)$ & $77.4(3)$ \\
\hline $\mathrm{N}(8)-\mathrm{Co}(2)-\mathrm{N}(5)$ & $93.0(3)$ & $96.1(3)$ & $\mathrm{N}(19)-\mathrm{Co}(2)-\mathrm{N}(18)$ & $85.6(3)$ & $83.2(3)$ \\
\hline $\mathrm{N}(8)-\mathrm{Co}(2)-\mathrm{N}(17)$ & $90.7(3)$ & $92.9(3)$ & $\mathrm{N}(20)-\mathrm{Co}(2)-\mathrm{N}(17)$ & $175.7(3)$ & $172.0(3)$ \\
\hline $\mathrm{N}(8)-\mathrm{Co}(2)-\mathrm{N}(18)$ & $92.3(3)$ & $93.4(3)$ & $\mathrm{N}(20)-\mathrm{Co}(2)-\mathrm{N}(18)$ & $94.7(3)$ & $97.0(3)$ \\
\hline $\mathrm{N}(8)-\mathrm{Co}(2)-\mathrm{N}(19)$ & $174.3(4)$ & $169.0(4)$ & $\mathrm{N}(20)-\mathrm{Co}(2)-\mathrm{N}(19)$ & $83.2(4)$ & $76.9(4)$ \\
\hline $\mathrm{N}(6)-\mathrm{Co}(3)-\mathrm{N}(21)$ & $94.4(3)$ & $94.9(3)$ & $\mathrm{N}(9)-\mathrm{Co}(3)-\mathrm{N}(23)$ & $176.4(3)$ & $172.5(3)$ \\
\hline $\mathrm{N}(6)-\mathrm{Co}(3)-\mathrm{N}(22)$ & $174.8(3)$ & $170.1(3)$ & $\mathrm{N}(9)-\mathrm{Co}(3)-\mathrm{N}(24)$ & $95.3(3)$ & $96.3(3)$ \\
\hline $\mathrm{N}(6)-\mathrm{Co}(3)-\mathrm{N}(23)$ & $88.2(3)$ & $88.3(3)$ & $\mathrm{N}(21)-\mathrm{Co}(3)-\mathrm{N}(22)$ & $81.2(3)$ & $76.1(3)$ \\
\hline $\mathrm{N}(6)-\mathrm{Co}(3)-\mathrm{N}(24)$ & $95.8(3)$ & $100.7(3)$ & $\mathrm{N}(21)-\mathrm{Co}(3)-\mathrm{N}(23)$ & $89.9(3)$ & $88.7(3)$ \\
\hline $\mathrm{N}(9)-\mathrm{Co}(3)-\mathrm{N}(21)$ & $93.6(3)$ & $98.7(3)$ & $\mathrm{N}(23)-\mathrm{Co}(3)-\mathrm{N}(22)$ & $89.0(3)$ & $87.2(3)$ \\
\hline $\mathrm{N}(9)-\mathrm{Co}(3)-\mathrm{N}(22)$ & $92.2(3)$ & 93.3(3) & $\mathrm{N}(24)-\mathrm{Co}(3)-\mathrm{N}(21)$ & $166.3(3)$ & $157.8(3)$ \\
\hline \multirow[t]{2}{*}{$\mathrm{N}(9)-\mathrm{Co}(3)-\mathrm{N}(6)$} & $90.8(3)$ & $92.3(3)$ & $\mathrm{N}(24)-\mathrm{Co}(3)-\mathrm{N}(22)$ & $88.1(3)$ & $86.8(3)$ \\
\hline & & & $\mathrm{N}(24)-\mathrm{Co}(3)-\mathrm{N}(23)$ & $81.3(3)$ & $76.2(3)$ \\
\hline $\mathrm{C}(1)-\mathrm{Fe}(1)-\mathrm{C}(2)$ & $87.9(4)$ & $88.6(4)$ & $\mathrm{C}(4)-\mathrm{Fe}(1)-\mathrm{C}(3)$ & $177.4(4)$ & $179.3(4)$ \\
\hline $\mathrm{C}(1)-\mathrm{Fe}(1)-\mathrm{C}(3)$ & $89.8(4)$ & $91.0(4)$ & $\mathrm{C}(5)-\mathrm{Fe}(1)-\mathrm{C}(2)$ & $89.3(4)$ & $89.2(4)$ \\
\hline $\mathrm{C}(1)-\mathrm{Fe}(1)-\mathrm{C}(4)$ & $87.6(4)$ & $88.8(4)$ & $\mathrm{C}(5)-\mathrm{Fe}(1)-\mathrm{C}(4)$ & $88.7(4)$ & $88.4(4)$ \\
\hline $\mathrm{C}(1)-\mathrm{Fe}(1)-\mathrm{C}(5)$ & $175.3(4)$ & $176.5(4)$ & $\mathrm{C}(5)-\mathrm{Fe}(1)-\mathrm{C}(3)$ & $94.0(4)$ & $91.8(4)$ \\
\hline $\mathrm{C}(6)-\mathrm{Fe}(1)-\mathrm{C}(1)$ & $96.4(4)$ & $95.8(4)$ & $\mathrm{C}(6)-\mathrm{Fe}(1)-\mathrm{C}(2)$ & $175.5(4)$ & $175.7(4)$ \\
\hline $\mathrm{C}(2)-\mathrm{Fe}(1)-\mathrm{C}(3)$ & $91.7(4)$ & $91.7(4)$ & $\mathrm{C}(6)-\mathrm{Fe}(1)-\mathrm{C}(3)$ & $87.0(4)$ & $87.9(4)$ \\
\hline $\mathrm{C}(2)-\mathrm{Fe}(1)-\mathrm{C}(4)$ & $88.3(4)$ & $87.7(4)$ & $\mathrm{C}(6)-\mathrm{Fe}(1)-\mathrm{C}(4)$ & $93.3(4)$ & $92.8(4)$ \\
\hline$C(7)-\mathrm{Fe}(2)-\mathrm{C}(11)$ & $86.6(5)$ & $88.0(4)$ & $\mathrm{C}(6)-\mathrm{Fe}(1)-\mathrm{C}(5)$ & $86.6(4)$ & $86.5(4)$ \\
\hline $\mathrm{C}(8)-\mathrm{Fe}(2)-\mathrm{C}(7)$ & $86.2(4)$ & $86.7(4)$ & $\mathrm{C}(10)-\mathrm{Fe}(2)-\mathrm{C}(9)$ & $85.9(4)$ & $86.4(4)$ \\
\hline $\mathrm{C}(8)-\mathrm{Fe}(2)-\mathrm{C}(9)$ & $89.4(4)$ & $88.6(4)$ & $\mathrm{C}(10)-\mathrm{Fe}(2)-\mathrm{C}(11)$ & $91.1(4)$ & $92.0(5)$ \\
\hline $\mathrm{C}(8)-\mathrm{Fe}(2)-\mathrm{C}(11)$ & $93.6(4)$ & $93.1(4)$ & $\mathrm{C}(12)-\mathrm{Fe}(2)-\mathrm{C}(7)$ & $175.6(4)$ & $175.4(4)$ \\
\hline $\mathrm{C}(9)-\mathrm{Fe}(2)-\mathrm{C}(7)$ & $91.3(4)$ & $90.3(4)$ & $\mathrm{C}(12)-\mathrm{Fe}(2)-\mathrm{C}(8)$ & $90.5(4)$ & $88.9(4)$ \\
\hline $\mathrm{C}(9)-\mathrm{Fe}(2)-\mathrm{C}(11)$ & $176.2(4)$ & $177.6(4)$ & $\mathrm{C}(12)-\mathrm{Fe}(2)-\mathrm{C}(9)$ & $91.5(4)$ & $90.8(4)$ \\
\hline $\mathrm{C}(10)-\mathrm{Fe}(2)-\mathrm{C}(7)$ & $94.8(4)$ & $93.9(4)$ & $\mathrm{C}(12)-\mathrm{Fe}(2)-\mathrm{C}(10)$ & $90.8(5)$ & $90.5(4)$ \\
\hline $\mathrm{C}(10)-\mathrm{Fe}(2)-\mathrm{C}(8)$ & $175.2(4)$ & $175.0(4)$ & $\mathrm{C}(12)-\mathrm{Fe}(2)-\mathrm{C}(11)$ & $88.8(4)$ & $91.0(5)$ \\
\hline
\end{tabular}


Table S5. Average M-L bond distances $(\AA)$ for $\mathbf{1}$ at representative temperatures before $\left(\mathbf{1}^{\mathbf{a}}\right.$ and $\left.\mathbf{1}^{\mathrm{c}}\right)$ and after $\left(\mathbf{1}^{\mathbf{h}}\right.$ and $\left.\mathbf{1}^{\mathbf{i}}\right)$ the onset of the structural transformation at the Co sites.

\begin{tabular}{|c|c|c|c|c|}
\hline Connectivity & $\begin{array}{c}10^{\mathrm{a}} \\
T=30(2) \mathrm{K}\end{array}$ & $\begin{aligned} & 10^{\mathrm{C}} \\
T= & 110(2) \mathrm{K}\end{aligned}$ & $\begin{aligned} & 10^{\mathrm{h}} \\
T= & 220(2) \mathrm{K}\end{aligned}$ & $\begin{array}{c}10^{\mathrm{i}} \\
T=293(2) \mathrm{K}\end{array}$ \\
\hline $\mathrm{Co}(1)-\mathrm{N}$ & $2.08[5]$ & $2.12[5]$ & $2.13[4]$ & $2.10[5]$ \\
\hline $\mathrm{Co}(2)-\mathrm{N}$ & $1.96[3]$ & $1.96[3]$ & $2.08[4]$ & $2.09[4]$ \\
\hline $\mathrm{Co}(3)-\mathrm{N}$ & $1.98[4]$ & $1.98[4]$ & $2.09[4]$ & $2.10[5]$ \\
\hline $\mathrm{Fe}(1)-\mathrm{C}$ & $1.89[2]$ & $1.92[1]$ & $1.932[9]$ & $1.91[1]$ \\
\hline $\mathrm{Fe}(1)-\mathrm{C}$ & $1.88[2]$ & $1.91[3]$ & $1.91[1]$ & $1.91[3]$ \\
\hline
\end{tabular}


Table S6. Selected bond distances ( $)$ for 2 .

\begin{tabular}{llll}
\hline \multicolumn{1}{c}{ Connectivity } & \multicolumn{3}{c}{ Connectivity } \\
\hline $\operatorname{Co}(1)-\mathrm{N}(8)$ & $1.85(1)$ & $\mathrm{Co}(2)-\mathrm{N}(15)$ & $1.91(1)$ \\
$\mathrm{Co}(1)-\mathrm{N}(10)$ & $1.88(1)$ & $\mathrm{Co}(2)-\mathrm{N}(13)$ & $1.92(1)$ \\
$\mathrm{Co}(1)-\mathrm{N}(3)$ & $1.91(1)$ & $\mathrm{Co}(2)-\mathrm{N}(20)$ & $1.92(1)$ \\
$\mathrm{Co}(1)-\mathrm{N}(21)$ & $1.93(1)$ & $\mathrm{Co}(3)-\mathrm{N}(12)$ & $1.85(1)$ \\
$\mathrm{Co}(1)-\mathrm{N}(7)$ & $1.93(1)$ & $\mathrm{Co}(3)-\mathrm{N}(4)$ & $1.86(1)$ \\
$\mathrm{Co}(1)-\mathrm{N}(6)$ & $1.94(1)$ & $\mathrm{Co}(3)-\mathrm{N}(2)$ & $1.92(1)$ \\
$\mathrm{Co}(2)-\mathrm{N}(22)$ & $1.86(1)$ & $\mathrm{Co}(3)-\mathrm{N}(27)$ & $1.92(1)$ \\
$\mathrm{Co}(2)-\mathrm{N}(23)$ & $1.87(1)$ & $\mathrm{Co}(3)-\mathrm{N}(37)$ & $1.94(1)$ \\
$\mathrm{Co}(2)-\mathrm{N}(5)$ & $1.89(1)$ & $\mathrm{Co}(3)-\mathrm{N}(11)$ & $1.96(1)$ \\
$\mathrm{Fe}(4)-\mathrm{C}(62)$ & $1.87(1)$ & $\mathrm{Fe}(5)-\mathrm{C}(19)$ & $1.88(1)$ \\
$\mathrm{Fe}(4)-\mathrm{C}(85)$ & $1.87(1)$ & $\mathrm{Fe}(5)-\mathrm{C}(70)$ & $1.89(1)$ \\
$\mathrm{Fe}(4)-\mathrm{C}(29)$ & $1.88(1)$ & $\mathrm{Fe}(5)-\mathrm{C}(102)$ & $1.90(2)$ \\
$\mathrm{Fe}(4)-\mathrm{C}(44)$ & $1.89(1)$ & $\mathrm{Fe}(5)-\mathrm{C}(66)$ & $1.91(1)$ \\
$\mathrm{Fe}(4)-\mathrm{C}(26)$ & $1.89(1)$ & $\mathrm{Fe}(5)-\mathrm{C}(31)$ & $1.91(1)$ \\
$\mathrm{Fe}(4)-\mathrm{C}(90)$ & $1.91(1)$ & $\mathrm{Fe}(5)-\mathrm{C}(58)$ & $1.94(1)$ \\
\hline
\end{tabular}


Table S7. Selected bond angles ( $\AA$ ) for 2.

\begin{tabular}{|c|c|c|c|}
\hline Connectivity & & Connectivity & \\
\hline $\mathrm{N}(8)-\mathrm{Co}(1)-\mathrm{N}(10)$ & $91.3(4)$ & $\mathrm{C}(62)-\mathrm{Fe}(4)-\mathrm{C}(85)$ & $88.4(6)$ \\
\hline $\mathrm{N}(8)-\mathrm{Co}(1)-\mathrm{N}(3)$ & $90.8(5)$ & $\mathrm{C}(62)-\mathrm{Fe}(4)-\mathrm{C}(29)$ & $92.3(6)$ \\
\hline $\mathrm{N}(10)-\mathrm{Co}(1)-\mathrm{N}(3)$ & $93.5(5)$ & $C(85)-F e(4)-C(29)$ & $87.7(6)$ \\
\hline $\mathrm{N}(8)-\mathrm{Co}(1)-\mathrm{N}(21)$ & $93.1(5)$ & $C(62)-F e(4)-C(44)$ & $88.1(5)$ \\
\hline $\mathrm{N}(10)-\mathrm{Co}(1)-\mathrm{N}(21)$ & $90.7(4)$ & $C(85)-F e(4)-C(44)$ & $174.5(6)$ \\
\hline $\mathrm{N}(3)-\mathrm{Co}(1)-\mathrm{N}(21)$ & $174.2(5)$ & $C(29)-F e(4)-C(44)$ & $88.2(5)$ \\
\hline $\mathrm{N}(8)-\mathrm{Co}(1)-\mathrm{N}(7)$ & $176.0(5)$ & $C(62)-F e(4)-C(26)$ & $177.4(5)$ \\
\hline $\mathrm{N}(10)-\mathrm{Co}(1)-\mathrm{N}(7)$ & $90.3(4)$ & $C(85)-F e(4)-C(26)$ & $94.2(6)$ \\
\hline $\mathrm{N}(3)-\mathrm{Co}(1)-\mathrm{N}(7)$ & $92.7(5)$ & $C(29)-F e(4)-C(26)$ & $87.7(5)$ \\
\hline $\mathrm{N}(21)-\mathrm{Co}(1)-\mathrm{N}(7)$ & $83.2(5)$ & $\mathrm{C}(44)-\mathrm{Fe}(4)-\mathrm{C}(26)$ & $89.3(5)$ \\
\hline $\mathrm{N}(8)-\mathrm{Co}(1)-\mathrm{N}(6)$ & $89.1(4)$ & $\mathrm{C}(62)-\mathrm{Fe}(4)-\mathrm{C}(90)$ & $89.8(6)$ \\
\hline $\mathrm{N}(10)-\mathrm{Co}(1)-\mathrm{N}(6)$ & $175.9(5)$ & $\mathrm{C}(85)-\mathrm{Fe}(4)-\mathrm{C}(90)$ & $92.9(6)$ \\
\hline $\mathrm{N}(3)-\mathrm{Co}(1)-\mathrm{N}(6)$ & $82.4(5)$ & $C(29)-F e(4)-C(90)$ & $177.9(6)$ \\
\hline $\mathrm{N}(21)-\mathrm{Co}(1)-\mathrm{N}(6)$ & $93.3(4)$ & $\mathrm{C}(44)-\mathrm{Fe}(4)-\mathrm{C}(90)$ & $91.4(6)$ \\
\hline $\mathrm{N}(7)-\mathrm{Co}(1)-\mathrm{N}(6)$ & $89.6(4)$ & $C(26)-F e(4)-C(90)$ & $90.2(6)$ \\
\hline $\mathrm{N}(22)-\mathrm{Co}(2)-\mathrm{N}(23)$ & $90.1(5)$ & $C(19)-F e(5)-C(70)$ & $90.6(6)$ \\
\hline $\mathrm{N}(22)-\mathrm{Co}(2)-\mathrm{N}(5)$ & $87.5(4)$ & $\mathrm{C}(19)-\mathrm{Fe}(5)-\mathrm{C}(102)$ & $88.9(6)$ \\
\hline $\mathrm{N}(23)-\mathrm{Co}(2)-\mathrm{N}(5)$ & $175.7(5)$ & $\mathrm{C}(70)-\mathrm{Fe}(5)-\mathrm{C}(102)$ & $179.2(6)$ \\
\hline $\mathrm{N}(22)-\mathrm{Co}(2)-\mathrm{N}(15)$ & $92.6(5)$ & $\mathrm{C}(19)-\mathrm{Fe}(5)-\mathrm{C}(66)$ & $178.7(6)$ \\
\hline $\mathrm{N}(23)-\mathrm{Co}(2)-\mathrm{N}(15)$ & $92.5(4)$ & $C(70)-F e(5)-C(66)$ & $90.7(5)$ \\
\hline $\mathrm{N}(5)-\mathrm{Co}(2)-\mathrm{N}(15)$ & $91.2(5)$ & $\mathrm{C}(102)-\mathrm{Fe}(5)-\mathrm{C}(66)$ & $89.9(5)$ \\
\hline $\mathrm{N}(22)-\mathrm{Co}(2)-\mathrm{N}(13)$ & $176.0(5)$ & $C(19)-F e(5)-C(31)$ & $90.8(5)$ \\
\hline $\mathrm{N}(23)-\mathrm{Co}(2)-\mathrm{N}(13)$ & $90.5(5)$ & $C(70)-F e(5)-C(31)$ & $88.2(5)$ \\
\hline $\mathrm{N}(5)-\mathrm{Co}(2)-\mathrm{N}(13)$ & $92.2(4)$ & $\mathrm{C}(102)-\mathrm{Fe}(5)-\mathrm{C}(31)$ & $91.2(6)$ \\
\hline $\mathrm{N}(15)-\mathrm{Co}(2)-\mathrm{N}(13)$ & $83.5(5)$ & $C(66)-F e(5)-C(31)$ & $89.0(5)$ \\
\hline $\mathrm{N}(22)-\mathrm{Co}(2)-\mathrm{N}(20)$ & $91.1(5)$ & $C(19)-F e(5)-C(58)$ & $91.7(6)$ \\
\hline $\mathrm{N}(23)-\mathrm{Co}(2)-\mathrm{N}(20)$ & $92.4(5)$ & $\mathrm{C}(70)-\mathrm{Fe}(5)-\mathrm{C}(58)$ & $92.8(5)$ \\
\hline $\mathrm{N}(5)-\mathrm{Co}(2)-\mathrm{N}(20)$ & $84.1(5)$ & $\mathrm{C}(102)-\mathrm{Fe}(5)-\mathrm{C}(58)$ & $87.8(6)$ \\
\hline $\mathrm{N}(15)-\mathrm{Co}(2)-\mathrm{N}(20)$ & $173.9(4)$ & $\mathrm{C}(66)-\mathrm{Fe}(5)-\mathrm{C}(58)$ & $88.5(6)$ \\
\hline $\mathrm{N}(13)-\mathrm{Co}(2)-\mathrm{N}(20)$ & $92.8(5)$ & $\mathrm{C}(31)-\mathrm{Fe}(5)-\mathrm{C}(58)$ & $177.3(6)$ \\
\hline $\mathrm{N}(12)-\mathrm{Co}(3)-\mathrm{N}(4)$ & $90.8(5)$ & $\mathrm{N}(12)-\mathrm{Co}(3)-\mathrm{N}(37)$ & $176.6(5)$ \\
\hline $\mathrm{N}(12)-\mathrm{Co}(3)-\mathrm{N}(2)$ & $89.7(4)$ & $\mathrm{N}(4)-\mathrm{Co}(3)-\mathrm{N}(37)$ & $90.5(4)$ \\
\hline $\mathrm{N}(4)-\mathrm{Co}(3)-\mathrm{N}(2)$ & $175.0(5)$ & $\mathrm{N}(2)-\mathrm{Co}(3)-\mathrm{N}(37)$ & $89.2(4)$ \\
\hline $\mathrm{N}(12)-\mathrm{Co}(3)-\mathrm{N}(27)$ & $89.7(5)$ & $\mathrm{N}(27)-\mathrm{Co}(3)-\mathrm{N}(37)$ & $93.4(5)$ \\
\hline $\mathrm{N}(4)-\mathrm{Co}(3)-\mathrm{N}(27)$ & $91.8(5)$ & $\mathrm{N}(12)-\mathrm{Co}(3)-\mathrm{N}(11)$ & $94.0(5)$ \\
\hline $\mathrm{N}(2)-\mathrm{Co}(3)-\mathrm{N}(27)$ & $83.2(5)$ & $\mathrm{N}(4)-\mathrm{Co}(3)-\mathrm{N}(11)$ & $89.5(4)$ \\
\hline $\mathrm{N}(27)-\mathrm{Co}(3)-\mathrm{N}(11)$ & $176.0(4)$ & $\mathrm{N}(2)-\mathrm{Co}(3)-\mathrm{N}(11)$ & $95.5(5)$ \\
\hline $\mathrm{N}(37)-\mathrm{Co}(3)-\mathrm{N}(11)$ & $82.9(5)$ & & \\
\hline
\end{tabular}


Table S8. Selected bond distances $(\AA)$ and angles $\left({ }^{\circ}\right)$ for 3.

\begin{tabular}{llll}
\hline \multicolumn{1}{c}{ Connectivity } & \multicolumn{2}{c}{ Connectivity } \\
\hline $\mathrm{Co}(1)-\mathrm{N}(1)$ & $1.922(4)$ & $\mathrm{Co}(2)-\mathrm{N}(4)$ & $1.949(4)$ \\
$\mathrm{Co}(1)-\mathrm{N}(2)$ & $1.928(3)$ & $\mathrm{Co}(2)-\mathrm{N}(5)$ & $1.919(3)$ \\
$\mathrm{Co}(1)-\mathrm{N}(11)$ & $1.863(4)$ & $\mathrm{Co}(2)-\mathrm{N}(23)$ & $1.879(3)$ \\
$\mathrm{Fe}(1)-\mathrm{C}(11)$ & $1.902(5)$ & $\mathrm{Fe}(2)-\mathrm{C}(23)$ & $1.901(4)$ \\
$\mathrm{Fe}(1)-\mathrm{C}(12)$ & $1.918(5)$ & $\mathrm{Fe}(2)-\mathrm{C}(24)$ & $1.914(5)$ \\
& & & \\
$\mathrm{N}(2)-\mathrm{Co}(1)-\mathrm{N}(1)$ & $82.8(2)$ & $\mathrm{N}(5)-\mathrm{Co}(2)-\mathrm{N}(4)$ & $82.8(1)$ \\
$\mathrm{N}(11)-\mathrm{Co}(1)-\mathrm{N}(1)$ & $88.7(2)$ & $\mathrm{N}(23)-\mathrm{Co}(2)-\mathrm{N}(4)$ & $177.2(1)$ \\
$\mathrm{N}(11)-\mathrm{Co}(1)-\mathrm{N}(2)$ & $89.1(2)$ & $\mathrm{N}(23)-\mathrm{Co}(2)-\mathrm{N}(5)$ & $94.5(1)$ \\
$\mathrm{C}(11)-\mathrm{Fe}(1)-\mathrm{C}(12)$ & $88.0(2)$ & $\mathrm{C}(24)-\mathrm{Fe}(2)-\mathrm{C}(23)$ & $86.6(2)$ \\
\hline
\end{tabular}


Figure S1. Mass spectrum of $\left\{\left[\mathrm{Co}(\text { tmphen })_{2}\right]_{3}\left[\mathrm{Fe}(\mathrm{CN})_{6}\right]_{2}\right\}(\mathbf{1})$ in $\mathrm{MeOH}$ over the range $\mathrm{m} / \mathrm{z}$ 600-1600 amu. Inset: Mass spectrum at higher mass ranges illustrating the coordination of $\mathrm{H}^{+}, \mathrm{Na}^{+}$, and $\mathrm{K}^{+}$to $\mathbf{1}$ in $\mathrm{MeOH}$ and the expanded mass spectrum at $m / z$ $1009 \mathrm{amu}$.

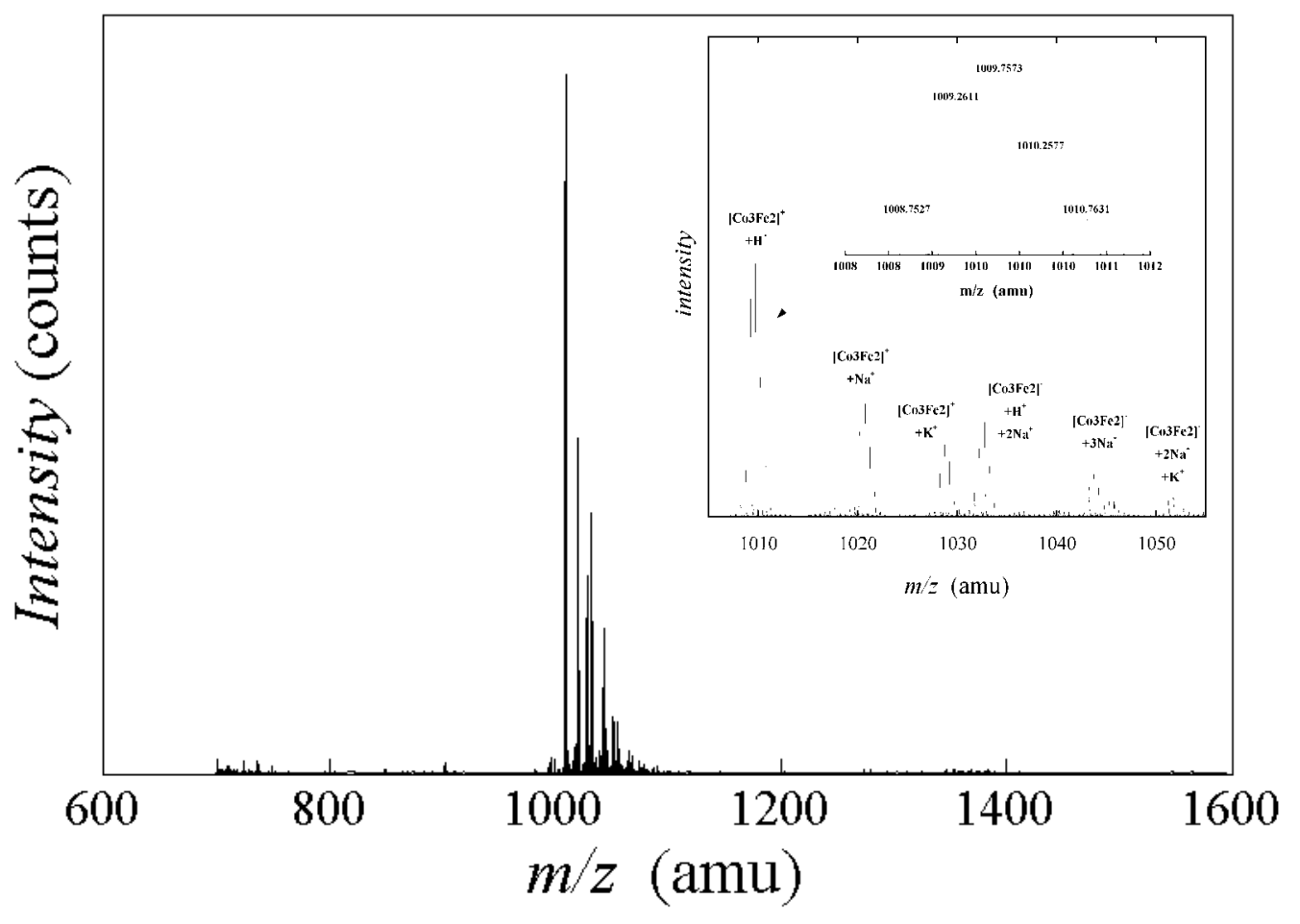


Figure S2. Packing diagram of two molecular units of 1 illustrating the $\pi-\pi$ contacts $(3.47 \AA$ ) of the tmphen ligands coordinated to the $\mathrm{Co}(1)$ ions.

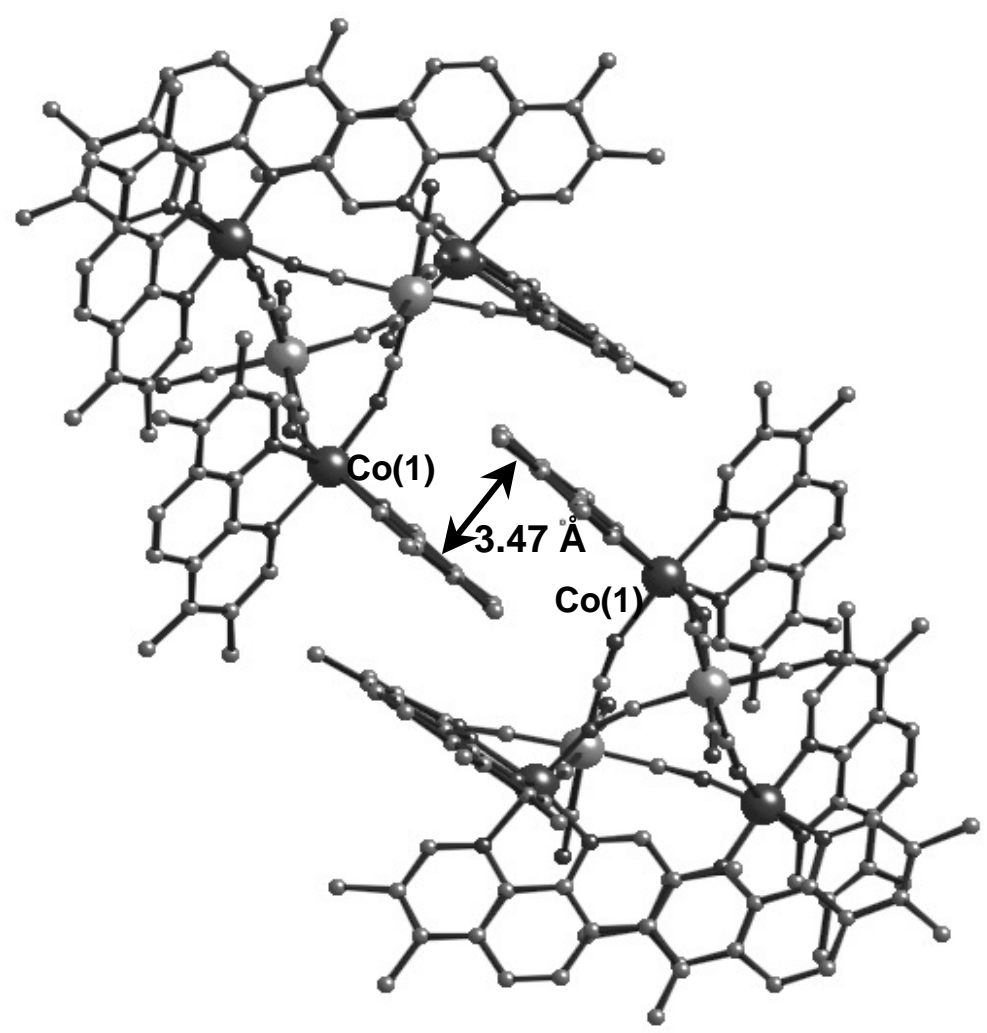


Figure S3. Packing diagram for 3 viewed along the $c$-axis. Intersitial $\mathrm{H}_{2} \mathrm{O}$ molecules have been omitted for the sake of clarity.

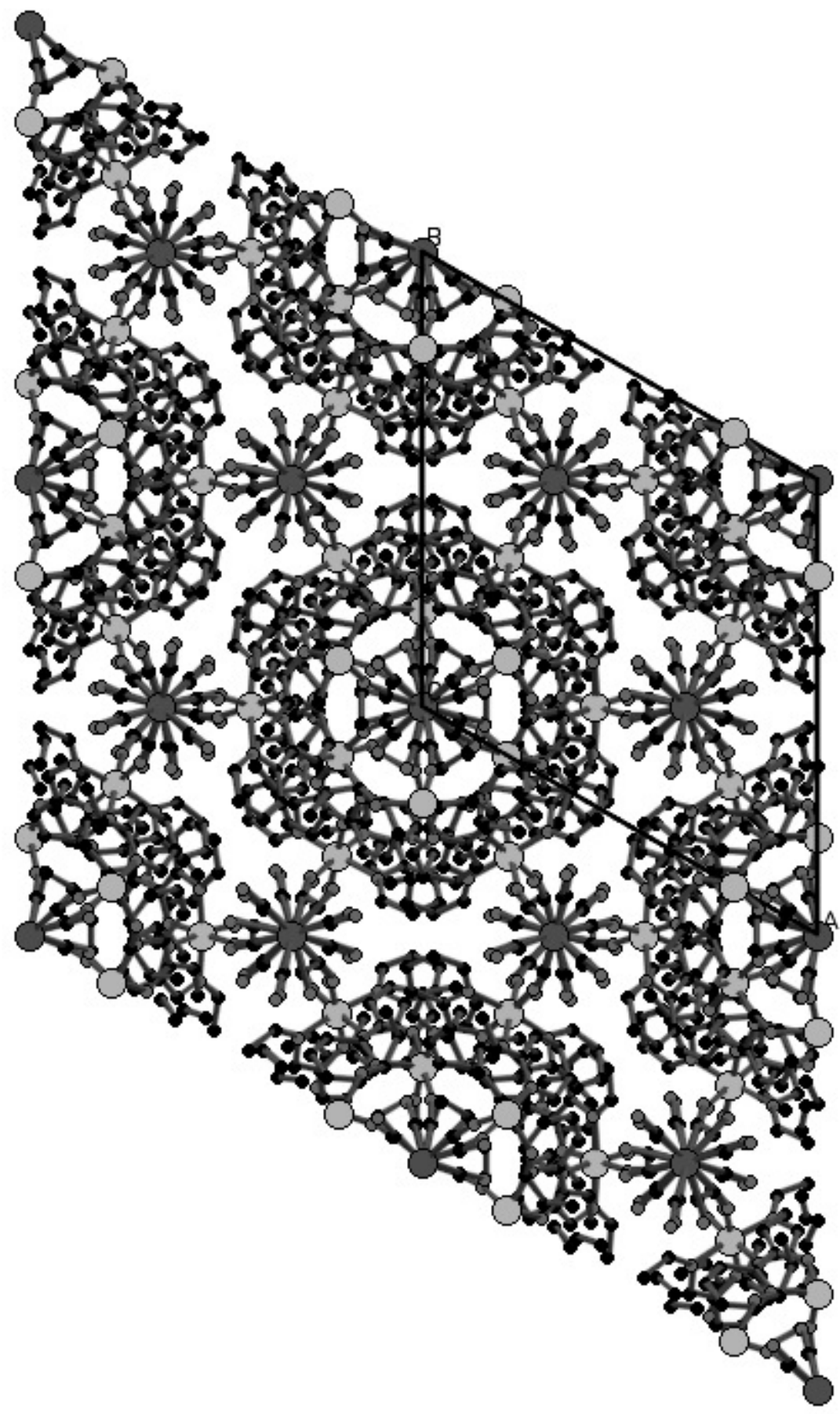


Figure S4. A depiction of the unit cell for 3 illustrating the locations of the $(\Lambda, \Lambda, \Lambda$ $\left\{\left[\mathrm{Co}(\mathrm{bpy})_{2}\right]_{3}\left[\mathrm{Fe}(\mathrm{CN})_{6}\right]_{2}\right\}^{+}$unit and the counterion, $\left[\mathrm{Fe}(\mathrm{CN})_{6}\right]^{3-}$. The $C_{3}$ axis defined by the Fe centers of the cation unit lies along the $(2 / 3,1 / 3, c)$ vector; the Fe ion of the anion, refined to $1 / 3$ occupancy, is disordered about the special position $(1 / 3,2 / 3,1 / 4)$. ( $H$ atoms, the $\left(\Delta, \Delta, \Delta-\left\{\left[\mathrm{Co}(\mathrm{bpy})_{2}\right]_{3}\left[\mathrm{Fe}(\mathrm{CN})_{6}\right]_{2}\right\}^{+}\right.$complex that lies along the $(1,0, c)$ vector, and intersitial solvent molecules have been omitted for clarity.)

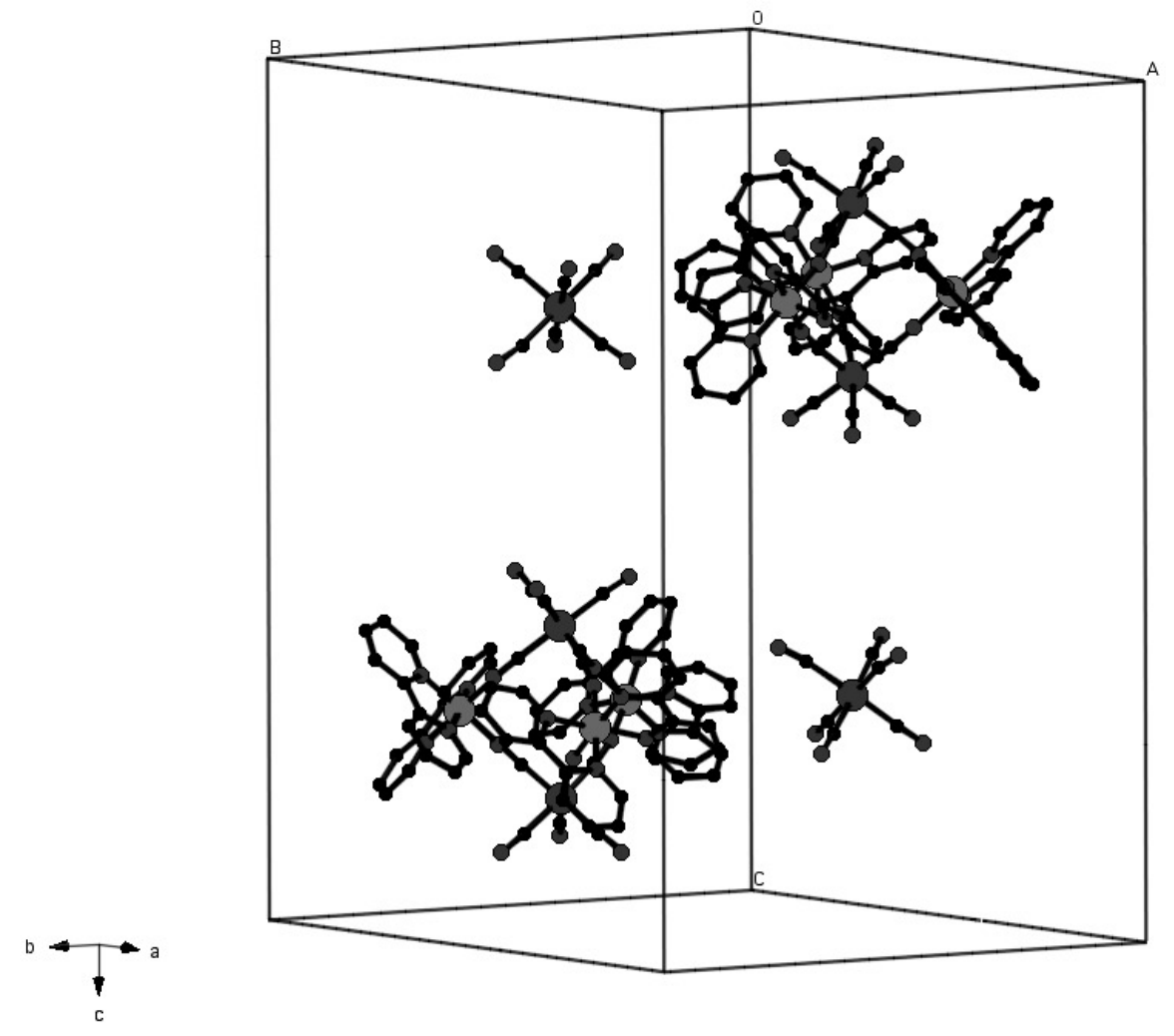


Figure S5. Mössbauer spectra and Fourier transforms of the spectra (shown above each corresponding spectrum) of the small red crystals of $\left\{\left[\mathrm{Co}(\text { tmphen })_{2}\right]_{3}\left[\mathrm{Fe}(\mathrm{CN})_{6}\right]_{2}\right\}$ (1) recorded in a magnetic field applied parallel to the observed $\gamma$-rays $(H=0.45 \mathrm{kG})$ at 30 $\mathrm{K}$ (a), $90 \mathrm{~K}$ (b), $110 \mathrm{~K}$ (c), $150 \mathrm{~K}$ (d), $190 \mathrm{~K}$ (e), and $220 \mathrm{~K}$ (f). Arrows mark the shoulder that is due to the left line of the doublet for LS Fe ${ }^{\mathrm{III}}$.

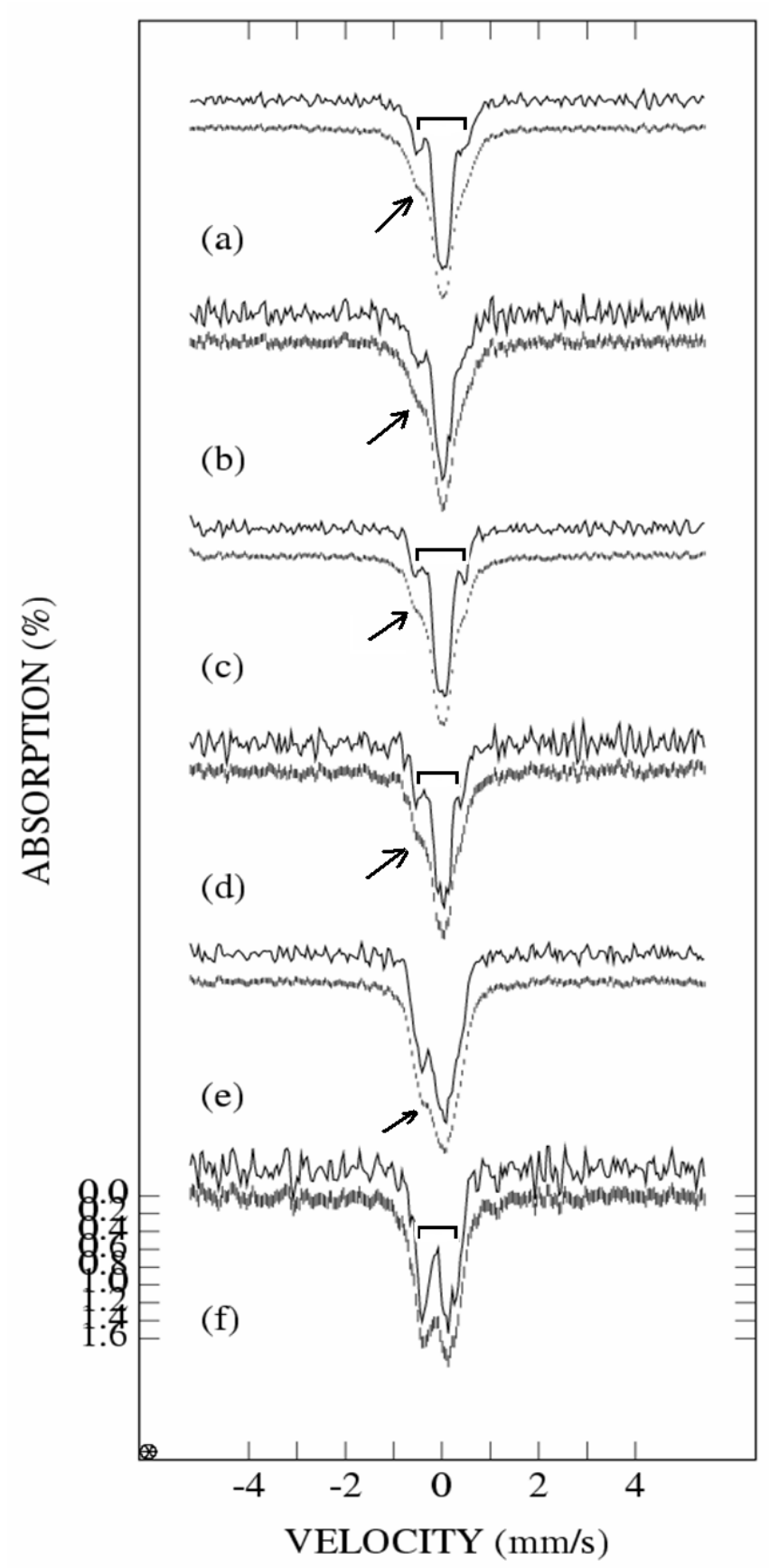


Figure S6. Mössbauer spectra of the red solid of $\left\{\left[\mathrm{Co}(\text { tmphen })_{2}\right]_{3}\left[\mathrm{Fe}(\mathrm{CN})_{6}\right]_{2}\right\}$ (1) after 1 (a), 5 (b), and10 days (c) under vacuum at 20 (top) and $100 \mathrm{~K}$ (bottom) $(H=0 \mathrm{G})$.
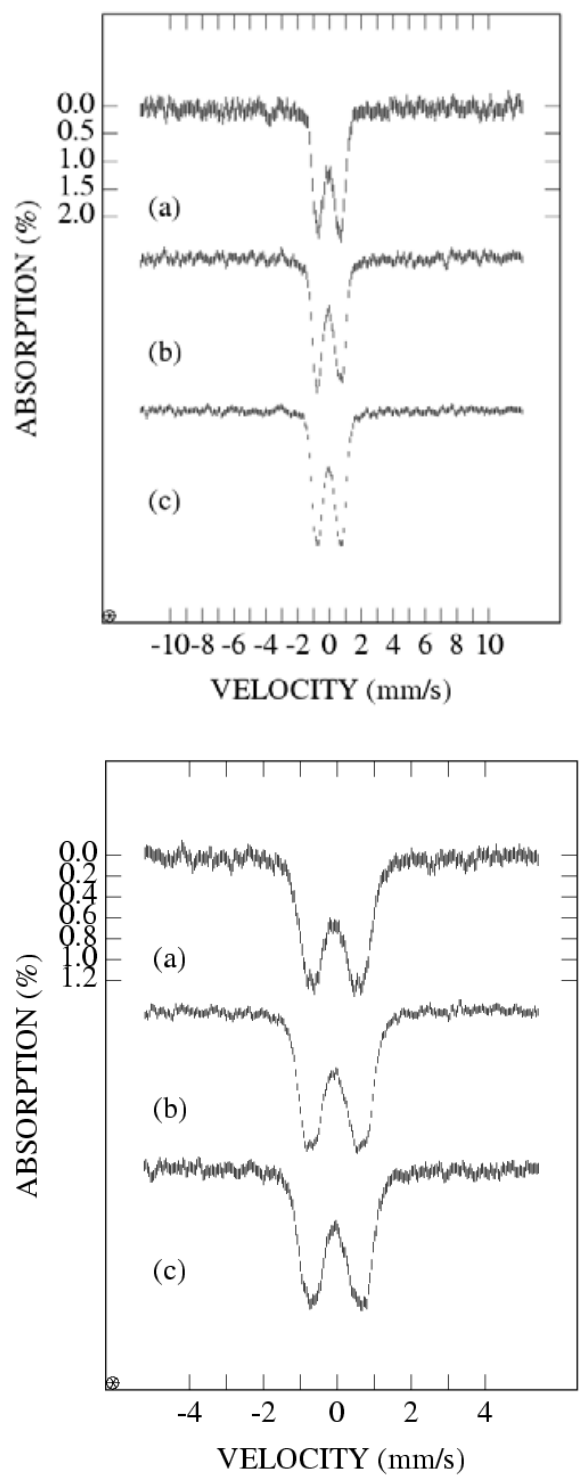
Figure S7. Mössbauer spectra of the red solid of $\left\{\left[\mathrm{Co}(\text { tmphen })_{2}\right]_{3}\left[\mathrm{Fe}(\mathrm{CN})_{6}\right]_{2}\right\}$ (1) after 1 (a), 5 (b), and10 days (c) under vacuum recorded in zero magnetic field at $T=4.2 \mathrm{~K}$.

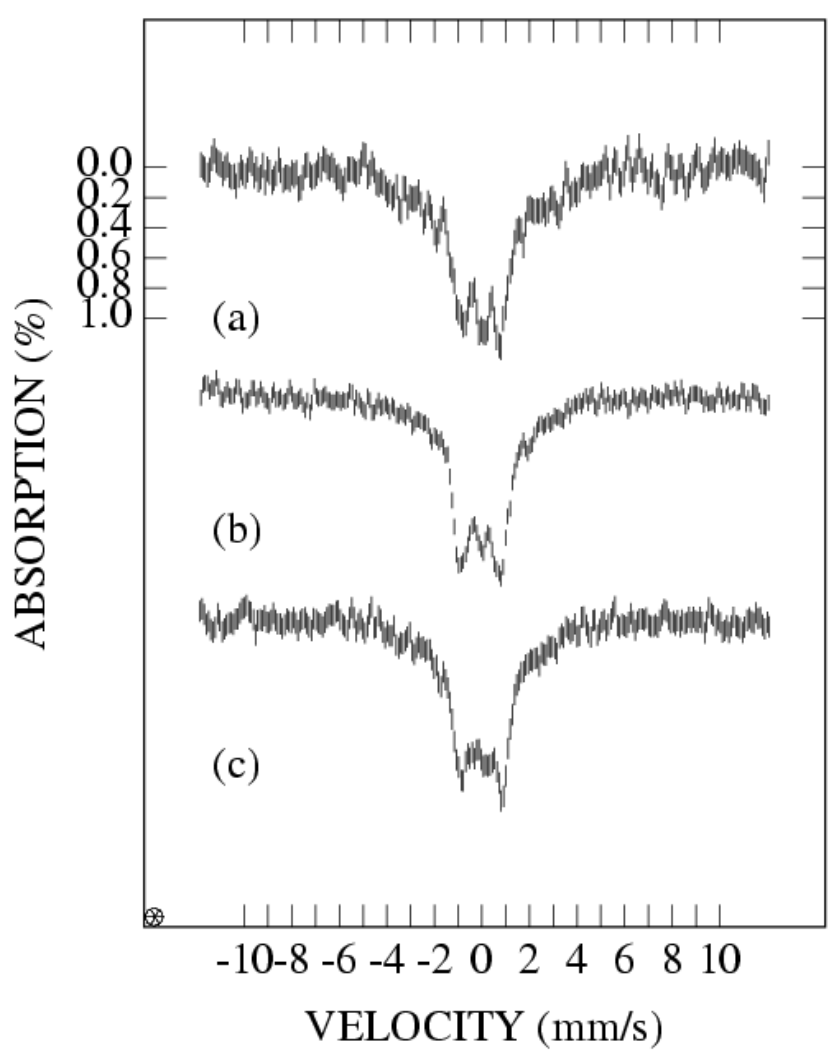


Figure S8. Plot of $\chi_{\mathrm{m}} T$ versus $T$ for 3 in the $2-300 \mathrm{~K}$ temperature range $(H=1000 \mathrm{G})$.

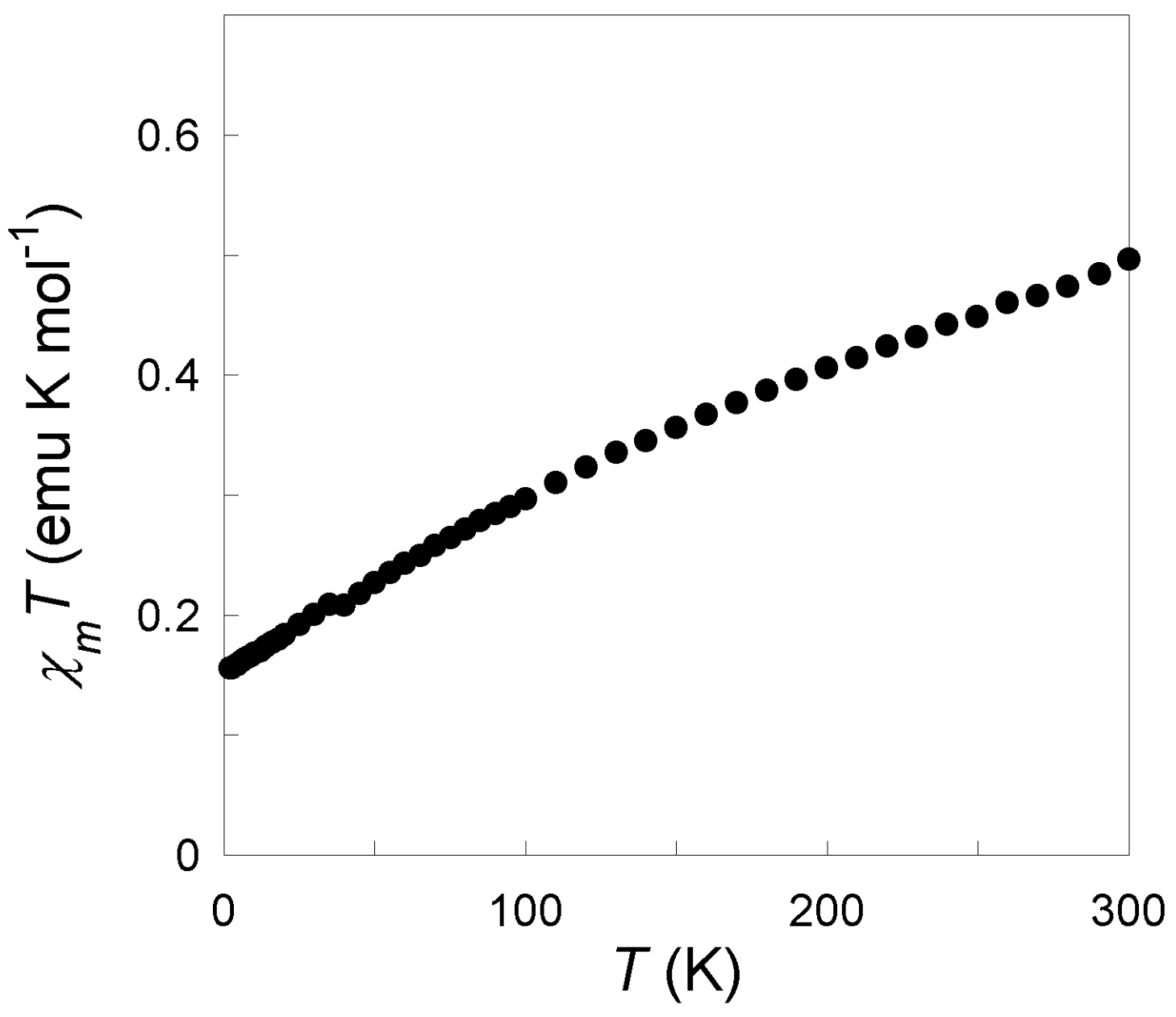

